\title{
A RESEARCH ON MOBILE APPLICATIONS FOR LOCATION TRACKING THROUGH WEB SERVER AND SHORT MESSAGES SERVICES (SMS)

\author{
SALMAN $^{1}$, WAHEED AHMAD $^{2}$, RIAZ ALI ${ }^{2}$, SALMAN SALEEM $^{3}$
}

${ }^{1}$ Department of Computer Science, Abdul Wali Khan, University Mardan, Khyber Pakhtunkhwa, Pakistan ${ }^{2}$ Department of Computer Science, Abdul Wali Khan, University Mardan, Khyber Pakhtunkhwa, Pakistan ${ }^{3}$ Department of Telecommunication Engineering University of Engineering \& Technology PeshawarKhyber Pakhtunkhwa, Pakistan Email: ${ }^{1}$ salman@awkum.edu.pk

Revised September 2015

ABSTRACT .This paper aims to develop an android application for android operating system (OS) platform that automatically send the current address location of the user to the server database and can also be sent periodically through SMS (short message services) to store mobile numbers by the user. Location of mobile device is in the form of latitude and longitude which is converted into full address by this application that includes country/state, city, and street number. In case of emergency the user can simply press the emergency button and the application will automatically send SMS alert including location address to the store mobile numbers that might be a police station or close relative. Another way to trace the user is the web server database which keeps updating the location address as long as the application is connected or last location address. The design shows how to implement and develop this app and has been tested on few mobile devices it well be tested on huge number of mobile devices later.

Keywords: Android Application, Short Message Services, Web Server, Web Services, Mobile Computing.

1. Introduction. Now a days with the rapid increase of Smartphone's devices, there users are also increasing as it becomes a major source of information. It is apparently showing the interest of users in mobile applications that provide storage, analysis and visualization of the information [1][12]. Most of smart phones equipped with computer processing ability are used to access network and use different applications developed to meet the user requirement via mobile communication network [18]. In last 07 years, different android Mobile applications have been developed including top paid and free apps also some focus on GPSGlobal Positioning System are developed [19].

In various research papers focus on Android Applications till March 2015, different methods are compared in order to use GPS for location tracking and routing[1][2][3]. Android-based mobile applications named as GPS phone tracker pro, Mobile Location Tracer, and Location Tracker provides location address of the user and famous applications on Google Play but these applications have no advance features but still more than 0.8 million people downloaded these applications[3][9]. Furthermore applications like Locate My Friends, Family Locator[8] and Family Locator \& Messaging[13] has also more than 0.6 million user downloaded history and are used for finding friends and family location but it has a lot of functionality complaints from users, which includes glitches and application crashing. Mobile Caller Location Tracker, All call Location Tracker and SMS Tracker has more than 0.5 million downloads and locates only user's location whose mobile phones are connected during call and short message service. There are also some famous mobile applications exit which tracks vehicle location like Vehicle Location Tracker[4], Find MyCar and Tracker these apps has more than 0.5 downloaded history but user complaints about the features of the apps. Some other applications are also designed with limited functionality to track lost devices e.g. Where My Droid [1], GPS Phone Tracker Pro and Mobile Location Tracker.

In this paper a GPS-based android mobile application is designed for GPS-enabled Smartphone's 
and is far richer in functionality as compare to the current commercial applications in the market, furthermore reliable methodology has been used to track down a specific location address and manipulate that location according to user requirements. The application track down current location of the user phone in the form of Latitude and Longitude convert it into full address then sent it to online server database using IMEI (International Mobile Station Equipment Identity) that can be used to find out user's last updated Location Address position which help in case of emergency.

Different tools have been used to develop our system which includes Java programming language, Android Studio platform, Android ADT and Android SDK for development of android application where code igniter, HTML and online database have been used for server side applet for providing online web services.

2. Block Diagram. The figure below shows the block diagram of android application that tracks the current location address of the user using GPS and sends it to an online web server automatically after a specific interval of time which keeps updating current location of the user. Sending location information to online database is a periodic process which performs without user intervention but the time period is adjustable.

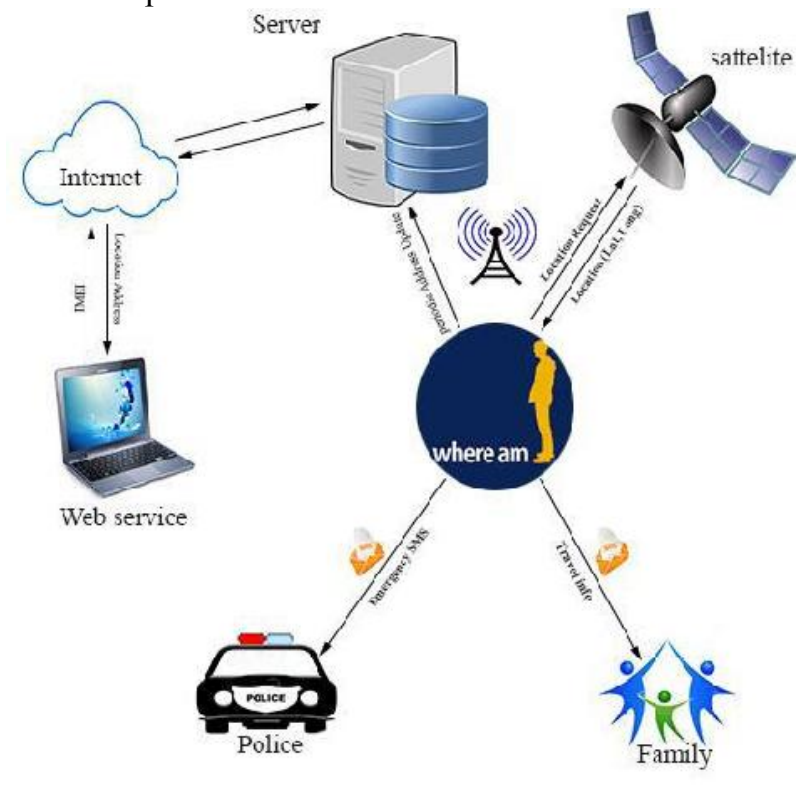

Figure: 1.1: Block diagram

In cases of lost or miss place of mobile the last updated address can be accessed via web service as shown in figure1.1. Furthermore application sends the location address of the user to stored mobile numbers periodically; these contact numbers are stored by the app user to inform their relative about travel. User can change the number and set the time-period accordingly. The last but not the least feature of the application is that, In case of any emergency the user can simply press the emergency button and the application will automatically send SMS alert info and including location address to store mobile numbers that might be nearest police station or close relative.

3. Results and Discussions: This whole software is divided into two major parts; one application is design for mobile phone and second for online web server. In scenario A, functionality of the app in mobile phone is presented while Scenario B presents the online web server.

Scenario A:Running the application on the first time after installation in mobile phone it display welcome screen for a while then it automatically open login activity to connect the user to online web server. It requires only password, as Username is the IMEI of the mobile phone, which the application picks automatically on startup. (*IMEI is hided). For new user Create Account option is provided to create the account, device IMEI is used as username. 


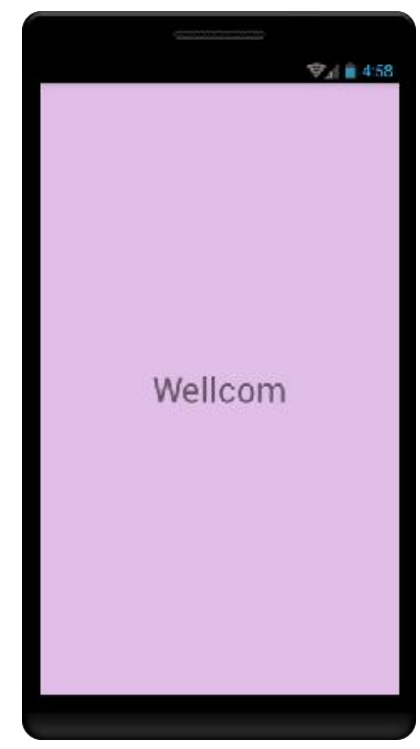

Figure 3.1: Welcome screen

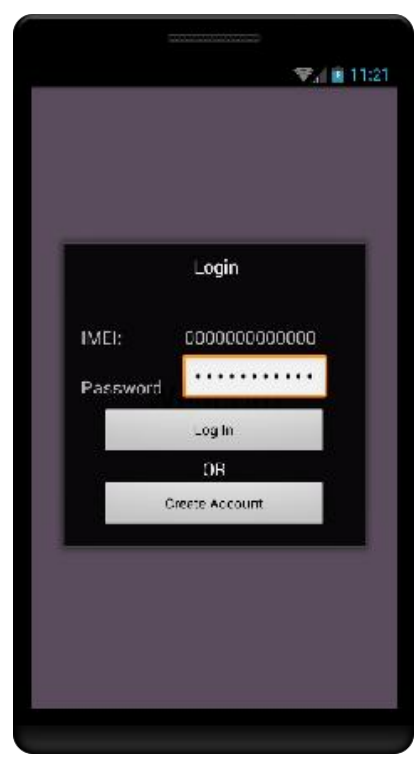

Figure 3.2: Login/ create account

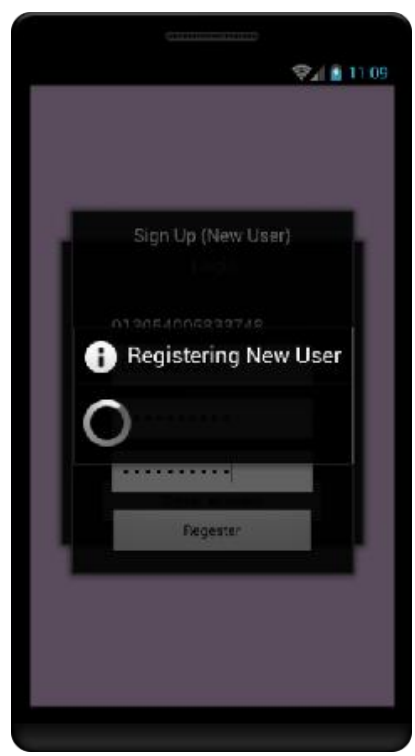

Figure 3.3: Registration new user

After successfully login into the system as shown in figure 3.4; the new user specify phone numbers to send periodic updates to inform about travel as well as police station number in case of emergency. The time period can also be managed to send periodic text messages to the phone numbers as shown in figure 3.7.User is recognized using provided credentials and the application feeds periodic location information to the web server accordingly.

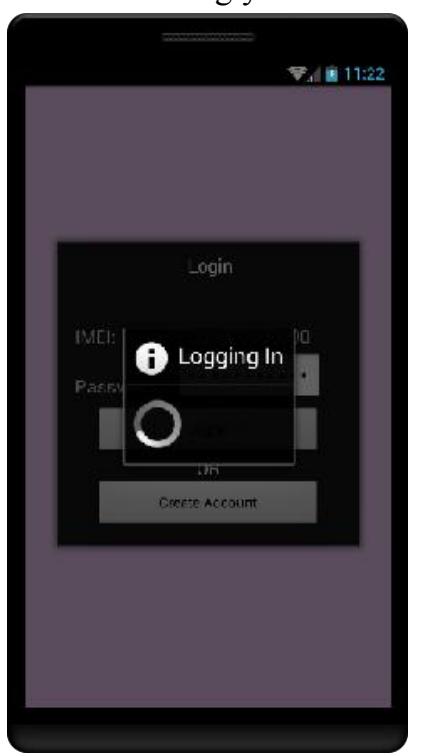

Figure 3.4: connection to server

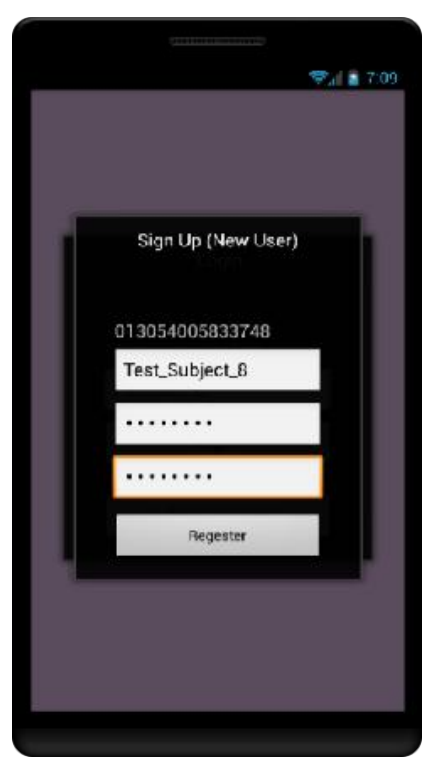

Figure 3.5: Sign up new user

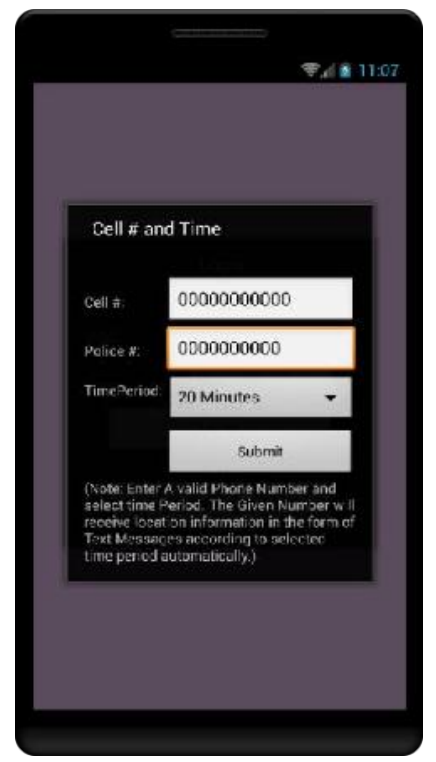

Figure 3.6: send address to cell \#

To access the current location address "where am I" button can be used as shown in figure3.8 which provides current location information including latitude, longitude and also full address in figure 3.9. Start sending button can be used to start sending location information to the stored mobile number as well as current location can be traced using Google maps. 


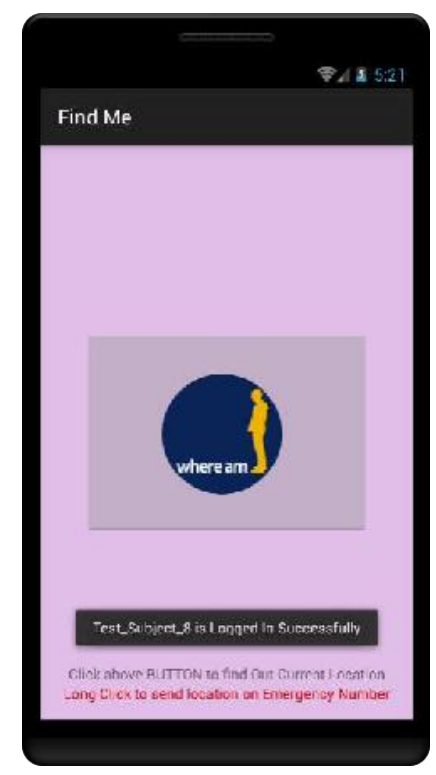

Figure 3.8: Where Iam Button

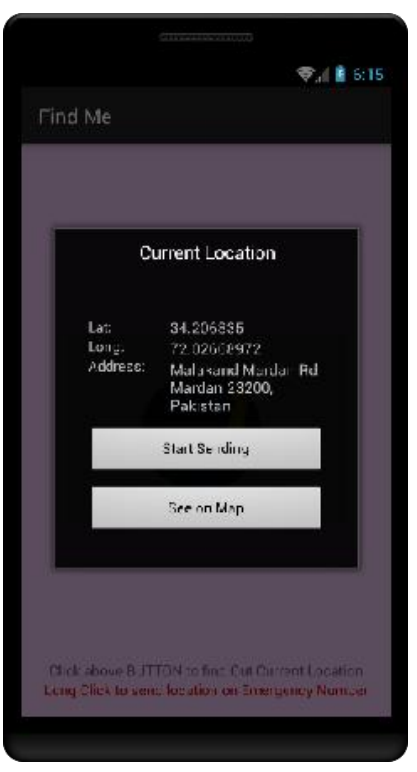

Figure 3.9: Current address info

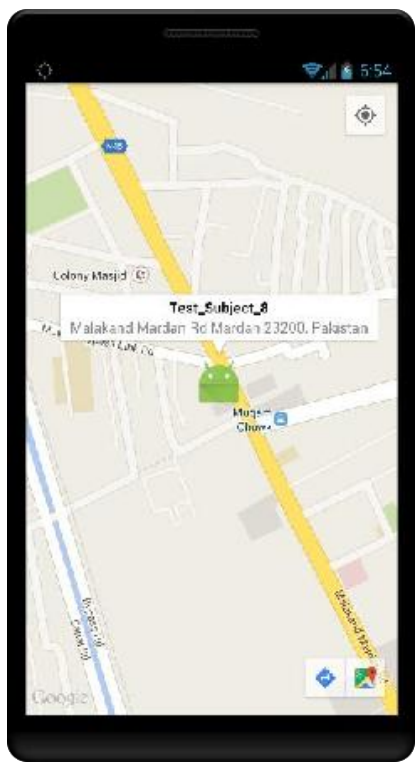

Figure 3.10: current location map

In Figure 3.11 shows that the current address can also be SMS to another contact number manually.
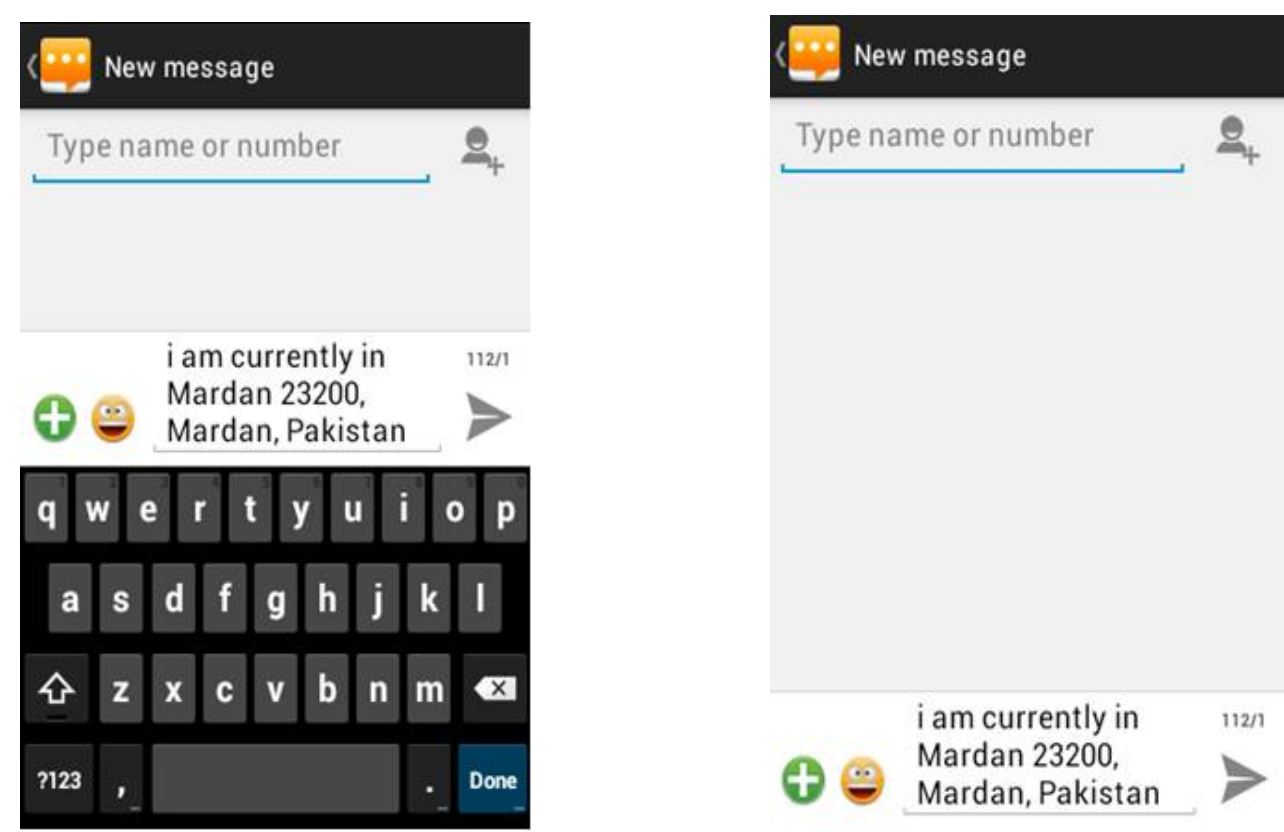

Figure 3.11: send address as SMS

To send location on Emergency bases long pressing the "Where am I" button sends current location address automatically to the stored number in the form of SMS (short message service) which contains an alert flag to inform the police station or any closed relative.

Scenario B: Online web Server application provides secure access to user location information using IMEI of device though web services. The online web services can be access only by registered user and web administrator. User cannot attempt on using fake credentials as the application picks up IMEI automatically 
on startup and create a connection to online web server and performs most of its automated functionalities, which includes updating database by providing latitude, longitude, address, date and time to the online server. The system validates both the username (which is IMEI) of device and password, provided by a user to authorize the user on both sides, application on client side which is Android Application and web services on server side.

Figure 3.13 shows the login screen for a user who has already been registered on mobile application. User needs to enter IMEI address of the device and password (used during sign up in figure 3.4). Figure 3.14 shows the stored information on server using periodic updates provided by the application. The given information includes latitude, longitude, address, time and date and a button in front of each update which forward user to maps view.

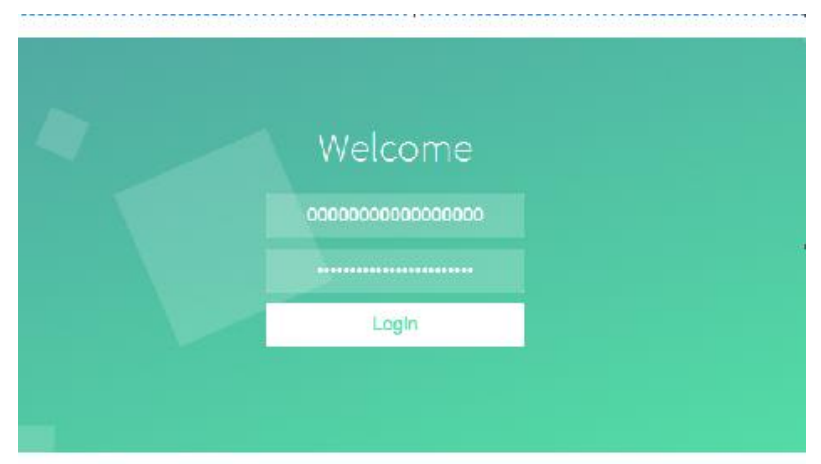

Figure 3.13: Server login screen

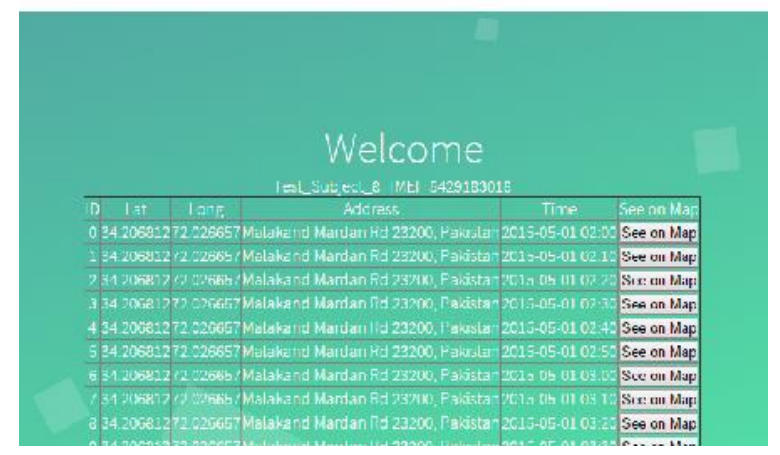

Figure 3.14: Periodic updates of address location

4. Conclusion. Smartphone's application changed the way of lives more than nothing especially android mobile applications. In more than 190 countries around the globe millions of Android mobile devices are founded and these numbers are growing day by day [14]. The main reason is the powerful mobile platform which helps the user to create different useful application from games to medical field (developer. Android, 2015). To conclude, thiswork aims to develop an android application for GPS enable smart phones which provide the current location address to the user and also update periodically to the online web server as far the user is connected otherwise the last updated location address will be store. In case of any emergency the user need one click and alert messages will be automatically transfer to local police station or close relative to inform about emergency and provide the user cur-rent location address. These contact numbers are also editable so the user can add or delete as many contact number as per user requirement.

As a future work, this application can be developed for different platform like windows, java and iOS.

\section{REFERENCES}

[1] AlienmanTechnologyLLC. (2015). Where My Droid. Retrieved from play.google.com: https://play.google.com/store/apps/details?id=com.alienmanfc6.wheresmyandroid\&hl=en 
[2] BruSd. (2015). Google Play store. Retrieved from Google Play Store website: https://play.google.com/store/apps/details?id=allcall.location.tracker\&hl=en

[3] CrazySoftech. (2015). Mobile Location Tracer. Retrieved from play.google.com: https://play.google.com/store/apps/details?id=com.crazyapps.mobilelocationtracker\&hl=en

[4] CrazySoftech. (2015). Vehicle Location Tracker. Retrieved from Google Play Store Website: https://play.google.com/store/apps/details?id=com.crazysoftech.vehiclelocationtracker\&hl=en

[5] Developer. Android. (2015). Android, the world's most popular mobile platform. Retrieved from developer.android.com: http://developer.android.com/about/index.html

[6] ELibera. (2014). Find my Car. Retrieved from Google Play Store Web site: https://play.google.com/store/apps/details?id=com.elibera.android.findmycar\&hl=en

[7] EMISMobile. (2014). Tracker. Retrieved from play.google.com: https://play.google.com/store/apps/details?id=sk.emis.tracker\&hl=en

[8] Familonet. (2015). Family Locator and messaging. Retrieved from play.google.ocm: https://play.google.com/store/apps/details?id=net.familo.android\&hl=en

[9] FamilySafetyProduction. (2015). GPS phone Tracker Pro. Retrieved from Play.Google.com: $\mathrm{https://play.google.com/store/apps/details?id=com.fsp.android.c \& hl=en}$

[10] FamilySafetyProduction. (2015). Locate My Friends. Retrieved from play.google.com: https://play.google.com/store/apps/details?id=com.fsp.android.friendlocator\&hl=en

[11] GizmoquipLLC. (2015). Google Play Store. Retrieved from Google Play Store Website: https://play.google.com/store/apps/details?id=com.gizmoquip.smstracker\&hl=en

[12] Hardy, B. (2014). The Big Nerd Ranch Guide.

[13] Life360. (2015). Family Locator. Retrieved from play.google.com: https://play.google.com/store/apps/details?id=com.life360.android.safetymapd\&hl=en

[14] Meier, R. (2012). Professional Android for Application Development. ISBN: 978-1-118-10227-5.

[15] Mobile Location Tracker. (2015, April 2). Retrieved from play.goog.com: https://play.google.com/store/apps/details?id=com.crazyapps.mobilelocationtracker\&hl=en

[16] OnexSoftech. (2015). Mobile Caller Location Tracker. Retrieved from play.google.com: https://play.google.com/store/apps/details?id=com.onexsoftech.callerlocation\&hl=en

[17] ZigurdMednieks, L. D. (2011). Programming Android.

[18] Salman, K., Ali, H., \& Saleem, S. (2015). Research on Auto Mobile-Pc Upload Images Application through Bluetooth Using Java.VAWKUM Transaction on Computer Sciences. Retrieved from http://vfast.org/journals/index.php/VTCS/article/view/356

[19] Saleem, S., Khan, I., khan, S., \& Rahman, A. (2015).Comparison of Cooperative Diversity Protocols in Various Relay Locations through Network Coding. VFAST Transactions on Software Engineering. Retrieved from http://vfast.org/journals/index.php/VTSE/article/view/357 